\title{
Luminescence of undoped LuAG and YAG crystals
}

\author{
V. Babin ${ }^{* 1}$, K. Blazek ${ }^{2}$, A. Krasnikov ${ }^{3}$, K. Nejezchleb ${ }^{2}$, M. Nikl ${ }^{4}$, T. Savikhina ${ }^{3}$, \\ and S. Zazubovich ${ }^{3}$ \\ ${ }^{1}$ Dept. of Condensed Matter, Utrecht University, Princetonplein 5, 3584 CC Utrecht, The Netherlands \\ ${ }^{2}$ CRYTUR Ltd., Palackeho 175, 51119 Turnov, Czech Republic \\ ${ }^{3}$ Institute of Physics, University of Tartu, Riia 142, 51014 Tartu, Estonia \\ ${ }^{4}$ Institute of Physics AS CR, Cukrovarnicka 10, 16253 Prague, Czech Republic
}

Received 11 July 2004, revised 24 August 2004, accepted 17 September 2004

Published online 20 January 2005

PACS 71.35.-y, 78.47.+p, 78.55.Hx, 78.60.-b

Emission and excitation spectra and decay kinetics are studied for the luminescence of undoped LuAG and YAG crystals. Two emission bands are located at $4.8 \mathrm{eV}$ and $3.95 \mathrm{eV}$ in YAG and $4.9 \mathrm{eV}$ and 3.65 $\mathrm{eV}$ in LuAG, excited in the $\mathrm{E}_{\text {exc }}>6.5 \mathrm{eV}$ energy region. They are both characterized by a relatively slow $(\mu \mathrm{s})$ decay time, and ascribed to the transitions from two different configurations of an exciton localized near an antisite defect $\left(\mathrm{Y}^{3+}{ }_{\mathrm{Al}}\right.$ or $\left.\mathrm{Lu}^{3+}{ }_{\mathrm{Al}}\right)$. The corresponding excitation band, peaking at $6.9 \mathrm{eV}$ in YAG and $7.2 \mathrm{eV}$ in $\mathrm{LuAG}$, is ascribed to the exciton perturbed by the antisite defect. Besides the two slow emissions, the weak fast (ns) emission of the self-trapped exciton, strongly overlapping with the high-energy emission band, is also observed. In $\mathrm{Ce}^{3+}$-doped garnets, the presence of the antisite defects results in a considerable decrease of energy transfer efficiency from the host lattice to $\mathrm{Ce}^{3+}$ ions. The possibilities of improvement of scintillation charactristics of this material are discussed.

() 2005 WILEY-VCH Verlag GmbH \& Co. KGaA, Weinheim

\section{Introduction}

$\mathrm{Ce}^{3+}$-doped yttrium-aluminium garnets $\mathrm{Y}_{3} \mathrm{Al}_{5} \mathrm{O}_{12}$ (YAG) and especially lutetium-aluminium garnets $\mathrm{Lu}_{3} \mathrm{Al}_{5} \mathrm{O}_{12}$ (LuAG) are effective scintillators, which show extremely high mechanical and chemical stability, high light yield and short luminescence decay times. In the luminescence spectrum of YAG:Ce and LuAG:Ce crystals, besides the $\mathrm{Ce}^{3+}$-related intense green and weak ultraviolet bands (see, e.g. [1, 2]), additional ultraviolet bands are observed, which are present also in undoped single crystals of YAG [312] and LuAG [2]. Defects, responsible for these bands, compete with $\mathrm{Ce}^{3+}$ in the energy transfer processes and reduce considerably the efficiency of energy transfer from the host lattice to $\mathrm{Ce}^{3+}$ ions [2].

The luminescence of undoped YAG crystals was studied in numerous papers (see, e.g., [1-13] and references therein). Under excitation in the exciton absorption region (near $7.0 \mathrm{eV}[3,4,8]$ ), the presence of two ultraviolet emission bands was reported. The high-energy band prevails at low temperatures. Its maximum position varies in various papers from $4.43 \mathrm{eV}$ [8] to $4.68 \mathrm{eV}$ [3], $4.9 \mathrm{eV}$ [4, 5, 7] and $4.95 \mathrm{eV}$ [6]. At RT a low-energy band is observed. Its maximum position varies from $4.2 \mathrm{eV}$ in [4-7] to $3.95 \mathrm{eV}$ in $[3,8]$. Quantum yields of both emissions are comparable and relatively high $(0.5$ in $[4,5] ; 0.1-0.5$ in [7]). In most papers (see, e.g., [4-8, 10-13] and references therein), the high-energy emission was ascribed to the self-trapped exciton (STE). In [4-7, 10], the low-energy emission was also connected with the STE. In [11-13] this band was ascribed to trapped excitons, and the antisite $\mathrm{Y}^{3+}{ }_{\mathrm{Al}}$ defects were considered as possible traps, the concentration of which can reach up to 0.5 at. \%. Characteristics of LuAG crystals were studied much less.

\footnotetext{
"Corresponding author: e-mail: V.Babin@phys.uu.nl, Phone: +49 408998 4526, Fax: +49 4089984475
} 
In the present paper, emission and excitation spectra and their dependences on temperature and defects concentration were studied at 80-295 K for two ultraviolet emission bands of undoped single crystals of LuAG and YAG under 6.0-11.5 eV excitation. Decay kinetics of luminescence was measured at 10-295 $\mathrm{K}$ under synchrotron excitation. The origin of the luminescence centers in these crystals is discussed.

\section{Experimental procedures, results and discussion}

Single crystals of LuAG and YAG were grown using the Czochralski method in molybdenum crucibles by CRYTUR Ltd. Two samples of LuAG with a small (no. 8150) and large (no. 8136) defects concentration (estimated from the comparison of their TSL glow peaks light sums) were examined.

The measurements under excitation in the energy range from $5.0 \mathrm{eV}$ to $11.5 \mathrm{eV}$ were carried out at a set-up with a vacuum monochromator VMR-2, hydrogen discharge open-capillary lamp and grating monochromator. Luminescence was detected by the photomultiplier FEU-39. Emission and excitation spectra were corrected for the experimental distortions, for the details see [2]. The spectra were measured under the same conditions therefore they can be compared also quantitatively. The high-energy emission was separated with a $257 \mathrm{~nm}(4.82 \mathrm{eV})$ interference filter, and the low-energy emission with a $357 \mathrm{~nm}$ $(3.47 \mathrm{eV}$ ) interference filter or with FS-6+BS-7 filters (transmission maximum near $3.8 \mathrm{eV}$ ).

Decay kinetics measurements at 10-295 K were performed at the HIGITI experimental station of HASYLAB at DESY, Hamburg, Germany. The excitation intensity was normalized to equal quantum intensities of synchrotron radiation falling onto a sample. The experimental setup is described in [14].

Luminescence characteristics of undoped YAG and LuAG crystals are similar. For YAG, they are similar to those reported in $[3-8,10]$. Under excitation in the energy region $\mathrm{E}_{\text {exc }}>6.5 \mathrm{eV}$, two emission bands are observed (Table 1). At low temperature, mainly the high-energy emission band, peaking at 4.9 $\mathrm{eV}$ in LuAG (Fig. 1a, curve 1) and at $4.8 \mathrm{eV}$ in YAG (Fig. 1b, curve 1), is present. The low-energy emission band, peaking at $3.65 \mathrm{eV}$ in $\mathrm{LuAG}$ and at $3.95 \mathrm{eV}$ in YAG, dominates at RT (curves 1'). The maximum intensities, positions and halfwidths of emission bands in two strongly different LuAG samples studied are close both at $85 \mathrm{~K}$ and at $300 \mathrm{~K}$ (Fig. 1a, compare solid and dotted lines). At $\mathrm{E}_{\mathrm{exc}}=7.7 \mathrm{eV}$, the intensities of both bands in LuAG are about 2 times larger than in YAG. At $80 \mathrm{~K}$ the intensity of the 4.9 $\mathrm{eV}$ emission in undoped LuAG crystals is only about 2.5 times larger than in the LuAG:Ce 0.06 mole \% crystal studied in [2]. It points to the fact that the efficiencies of energy transfer from the host lattice to $\mathrm{Ce}^{3+}$ ions and to the defects responsible for the $4.9 \mathrm{eV}$ emission are comparable.

Table 1 Maxima positions of excitation $\left(\mathrm{E}^{\mathrm{m}}{ }_{\text {exc }}\right)$ and emission $\left(\mathrm{E}_{\mathrm{em}}\right)$ bands and halfwidths (FWHM) of emission bands of YAG and LuAG crystals at $85 \mathrm{~K}$ and $295 \mathrm{~K}$. The temperature $\left(\mathrm{T}_{\mathrm{res}}\right)$ where the intensity redistribution between the two emission bands takes place.

\begin{tabular}{|c|c|c|c|c|c|c|c|}
\hline & $\mathrm{E}_{\mathrm{exc}}^{\mathrm{m}}, \mathrm{eV}$ & $\begin{array}{l}\mathrm{E}_{\mathrm{em}}, \mathrm{eV} \\
\mathrm{T}=85 \mathrm{~K}\end{array}$ & FWHM, eV & $\mathrm{E}_{\mathrm{exc}}^{\mathrm{m}}, \mathrm{eV}$ & $\begin{array}{l}E_{e m}, e V \\
T=295 K\end{array}$ & FWHM, eV & $\mathrm{T}_{\mathrm{res}}, \mathrm{K}$ \\
\hline YAG & 6.9 & 4.8 & 0.8 & 6.8 & 3.95 & 1.23 & 180 \\
\hline LuAG (8150) & 7.2 & 4.9 & 0.8 & 7.0 & 3.65 & 1.30 & 152 \\
\hline LuAG (8136) & 7.2 & 4.9 & 0.8 & 7.0 & 3.65 & 1.30 & 152 \\
\hline
\end{tabular}

Both emissions are excited only in the band-to-band transitions region and in the exciton absorption region. At LNT the emission intensity is maximal under excitation near $7.2 \mathrm{eV}$ in LuAG and $6.9 \mathrm{eV}$ in YAG (Fig. 1, curves 2). In each crystal the excitation band maximum of the emission studied is located between the excitation band of the STE emission in thin films (7.35 eV and $7.2 \mathrm{eV}$, respectively [13]) and the excitation band of the $\mathrm{Ce}^{3+}$ emission in LuAG:Ce and YAG:Ce (7.0 eV and $6.8 \mathrm{eV}$ [2]) which can be ascribed to an exciton perturbed by an $\mathrm{Ce}^{3+}$ ion. Excitation spectra of the high and low-energy emission bands are similar in shape within 80-300 K. At RT, the excitation spectra measured at opposite sides of the low-energy emission band of YAG and both LuAG crystals completely coincide. 


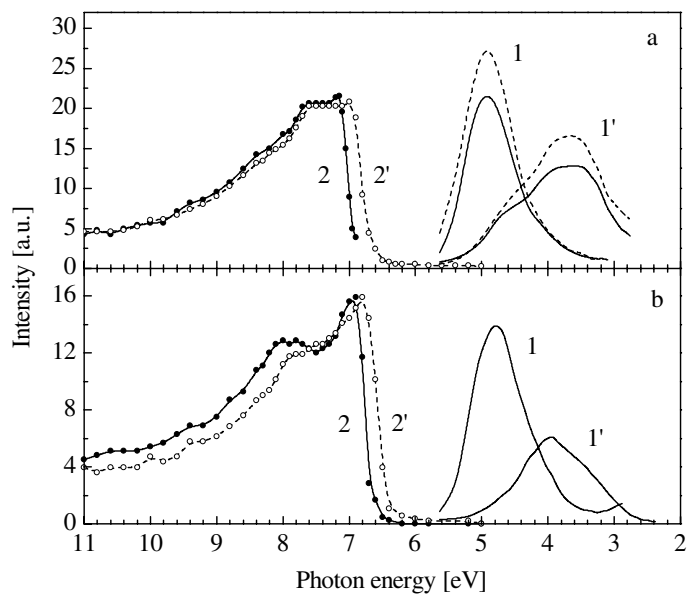

Fig. 1 Emission $\left(\mathrm{E}_{\mathrm{exc}}=7.7 \mathrm{eV}\right.$ ) (curves 1, 1') and normalized excitation (curves 2, 2') spectra measured at 85 $\mathrm{K}$ (curves 1, 2) and $295 \mathrm{~K}$ (curves 1', 2') for (a) LuAG and (b) YAG crystals. Emission spectra of two LuAG's (no. 8150 , solid line and no. 8136, dotted line) can be compared quantitatively.

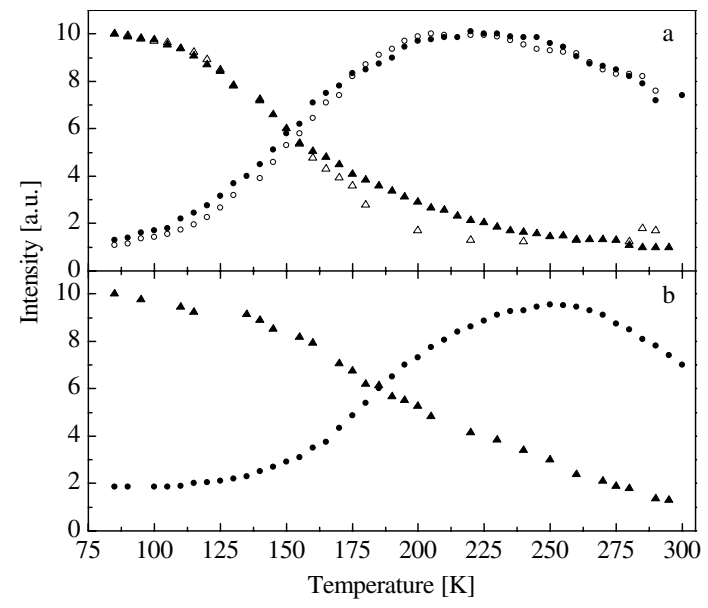

Fig. 2 Temperature dependences of emission intensities (normalized) measured for the high-energy (triangles) and low-energy (circles) emission bands (a) of two LuAG crystals (no.8136, open dots, no. 8150, filled dots) and (b) of YAG crystal. $\mathrm{E}_{\mathrm{exc}}=7.7 \mathrm{eV}$.

Thermally stimulated redistribution of emission intensities takes place near $152 \mathrm{~K}$ in LuAG (Fig. 2a) and near $180 \mathrm{~K}$ in YAG (Fig. 2b). The comparison of light sums of the emission bands measured at $85 \mathrm{~K}$ and 220-240 K allows the conclusion that both in YAG and LuAG crystals, the intensity redistribution occurs practically without losses (see also $[4,5]$ ).

In the YAG decay kinetics of both the emissions studied, slow ( $\mu \mathrm{s}$ ) components dominate (Fig. 3; see also $[4,9])$. Temperature dependences of their light sums are similar to those observed under the steady-

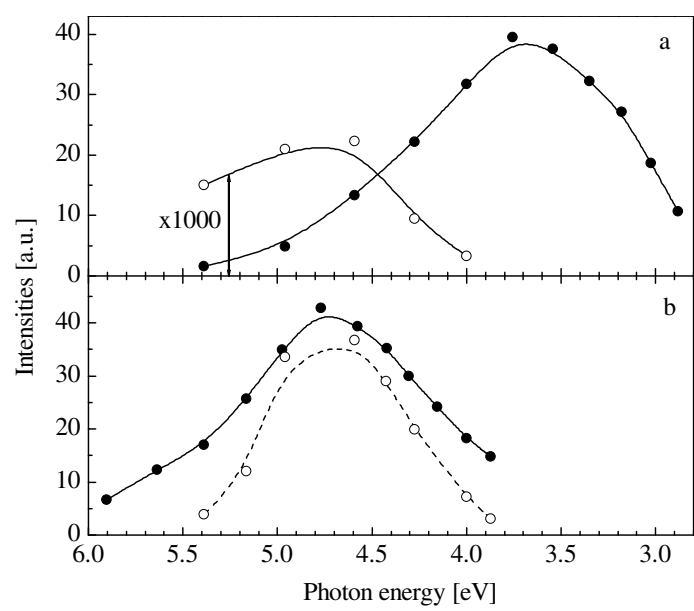

Fig. 3 Time-resolved emission spectra of (a) YAG (open points - fast component, filled points - slow component) and (b) for fast component of LuAG (no. 8136 by filled points and no. 8150 by open points) crystals at $295 \mathrm{~K}$.



Fig. 4 (a) Temperature dependences of decay times (points) and light sums (lines) of (a) the slow components of the high-energy (filled points; solid line) and low-energy (open points; dashed line) emission bands and (b) the fast component of the high-energy emission band (filled points, solid line) of YAG crystal. $\mathrm{E}_{\mathrm{exc}}=7.1 \mathrm{eV}$. 
state excitation (compare Figs. $2 \mathrm{~b}$ and $4 \mathrm{a}$ ). In YAG at $80 \mathrm{~K}$ the decay time of the slow component of the $4.8 \mathrm{eV}$ emission is about $3 \mu \mathrm{s}$. As the temperature increases, the decay time decreases down to about 0.4 $\mu \mathrm{s}$ at RT. The decay time of the $3.95 \mathrm{eV}$ emission is $\sim 10 \mu$ s at $150 \mathrm{~K}$ and $\sim 1 \mu$ s at RT (Fig. 4a). A much weaker, fast (2-4 ns) component is observed only in the region of the high-energy emission band. Its light sum at $80 \mathrm{~K}$ is by about 3 orders of magnitude smaller than the light sum of the slow component, it is independent of temperature up to $\sim 175 \mathrm{~K}$ and then rapidly decreases (Fig. 4b).

In LuAG crystals the situation is qualitatively similar to that of YAG described above. The decay time of the slow component of the $4.8 \mathrm{eV}$ emission is about $0.4 \mu \mathrm{s}$ at $10 \mathrm{~K}$. As the temperature increases, the decay time decreases down to about $0.2 \mu$ s at RT. The decay time of the $3.45 \mathrm{eV}$ emission is $\sim 0.6 \mu$ s at $80 \mathrm{~K}$. Weak fast (0.5-1.5 ns) components are observed in the regions of the high-energy emission band in the samples with small and large defects concentration. Its light sum at $80 \mathrm{~K}$ is by about 3 orders of magnitude smaller than the light sum of the slow component; the intensity increases up to $\sim 130 \mathrm{~K}$ and then gradually decreases. A much weaker, fast $(0.2-0.5 \mathrm{~ns})$ component is observed in the region of the low-energy emission band $(3.45 \mathrm{eV})$ in the sample with large defect concentration.

\section{Conclusions}

The data obtained allow the conclusion that both ultraviolet emission bands studied (Table 1) belong to the same centre. We assume that these emissions, related excitation bands (peaking at $6.9 \mathrm{eV}$ in YAG and near $7.2 \mathrm{eV}$ in $\mathrm{LuAG}$ ), and the dominating slow ( $\mu \mathrm{s})$ luminescence decay components arise from transitions related to two configurations of an exciton localized at $\mathrm{Y}^{3+}{ }_{\mathrm{Al}}\left(\mathrm{Lu}^{3+}{ }_{\mathrm{Al}}\right)$ antisite defects. Besides the two slow ultraviolet emissions, much weaker fast (ns) emission of the STE, overlapping with the high-energy emission band, is present in the single crystals. Mainly STE emission, peaking at $4.77 \mathrm{eV}$ in YAG and $4.96 \mathrm{eV}$ in $\mathrm{LuAG}$ and excited in the bands with maxima at $7.2 \mathrm{eV}$ and $7.35 \mathrm{eV}$, respectively, is observed in thin crystalline films of YAG and LuAG [13]. In the undoped single crystals, this emission is suppressed due to the presence of high concentration of antisite defects. As the presence of antisite defects leads to a strong decrease of the efficiency of energy transfer from the host lattice to the $\mathrm{Ce}^{3+}$ ions, for an improvement of scintillation characteristics of $\mathrm{Ce}^{3+}$-doped garnets the antisite defects should be removed.

Acknowledgements This work was supported by the NATO Science for Peace grant no. 973510 "SfPScintillators" and by the Estonian Science Foundation grant no. 5045.

\section{References}

[1] Y. Suzuki, T. Sakuma, and M. Hirai, Mater. Sci. Forum 239-241, 219 (1997).

[2] K. Blazek, A. Krasnikov, K. Nejezchleb, M. Nikl, T. Savikhina, and S. Zazubovich, phys. stat. sol. (b) 241 , 1124 (2004).

[3] N. S. Roose and N. A. Anisimov, Trudy Inst. Fiz. Akad. Nauk EstSSR 44, 163 (1975).

[4] A. I. Kuznetsov, V. N. Abramov, V. V. Mürk, and B. R. Namozov, Sov. Phys. Solid State 33, 1126 (1991).

[5] V. Mürk, A. Kuznetsov, B. Namozov, and K. Ismailov, Nucl. Instrum. Meth. B 91, 327 (1994).

[6] V. Mürk and N. Yaroshevich, J. Phys.: Condens. Matter 7, 5857 (1995).

[7] V. Mürk, Mater. Sci. Forum 239-241, 537 (1997).

[8] M. Kirm, A. Lushchik, Ch. Lushchik, and G. Zimmerer, in: Physics and Chemistry of Luminescence Materials, ed. C. Ronda et al., PV 99-40, The Electrochem. Soc. Proc. Ser. Pennington, NJ, 113 (2000).

[9] D. J. Robbins, B. Cockayne, J. L. Glasper, and B. Lent, J. Electrochem. Soc. 126, 1213 (1979).

[10] A. I. Kuznetsov, B. R. Namozov, and V. V. Mürk, Sov. Phys. Solid State 27, 1819 (1985).

[11] L. G. Volzhenskaya, Yu. V. Zorenko, N. I. Patsagan, and M. V. Pashkovsky, Opt. Spectr. 63, 790 (1987).

[12] Yu. V. Zorenko, V. Gorbenko, I. Konstankevych, V. Mikhailin, V. Kolobanov, and D. Spassky, Functional Mater. 9, 291 (2002).

[13] Y. Zorenko, A. Voloshinovskii, I. Konstankevych, V. Kolobanov, V. Mikhailin, and D. Spassky, Radiat. Meas. (in press) (2004).

[14] R. T. Wegh, H. Donker, A. Meijerink, R. J. Lamminmäki, and J. Hölsä, Phys. Rev. B 56, 13841 (1997). 